\title{
A multispectral VIS/SWIR sensor suite with integrated laser range finder
}

\author{
Mario Münzberg, Martin Hübner \\ Airbus DS Optronics GmbH, Carl-Zeiss Str. 22, 73447 Oberkochen, Germany \\ mario.muenzberg@airbusds-optronics.com
}

\begin{abstract}
:
For long range surveillance tasks the optics for the visible $(450 \mathrm{~nm}-700 \mathrm{~nm})$ and the short wave infrared (SWIR) spectral wavelength range $(900 \mathrm{~nm}-1700 \mathrm{~nm})$ are combined with the receiver optics of an integrated laser range finder (LRF) in a multispectral sensor suite. The incoming signal from the scene and the returned laser pulse are collected by the common entrance aperture. The front optics is broadband corrected from $450 \mathrm{~nm}-1700 \mathrm{~nm}$. Two beam splitters separate the incoming radiation to the appropriate sensors, such as HDTV CMOS chip, InGaAs focal plane array (FPA) and laser receiver diode. The visible spectrum is at first split up by a dichroic beam splitter and focused on the HDTV camera chip. The returned laser pulse is separated from the scene signal by a second beam splitter and focused on the laser receiver diode of the integrated LRF. The alignment of the LRF transmitter with respect to the line of sight of the SWIR camera can be adjusted by integrated wedges. The two images in the visible and the SWIR spectral range match in focus and field of view (FOV) over the full zoom range of a factor of 11 . The SWIR camera has a resolution of $640 \times 512$ pixels. The HDTV camera provides a resolution of $1920 \times 1080$. In the SWIR path the F-number is adapted to the corresponding chip dimensions defined by the pitch. For a 640 x 512 InGaAs FPA with $20 \mu \mathrm{m}$ pitch the $\mathrm{F \#}$ is $\mathrm{F} / 7$, for a $640 \times 512 \mathrm{InGaAs} F P A$ with $15 \mu \mathrm{m}$ pitch the optics adapts to $\mathrm{F} / 5.25$, whereas the $\mathrm{F \#}$ is F/2.6 for the visible path .
\end{abstract}

Key words: : Short wave infrared, multispectral, laser rangefinder, InGaAs, CMOS, continuous zoom

\section{Introduction}

Observation of far-off located targets under daylight conditions with CMOS or CCD based cameras with high focal length optics is in most cases limited by atmospheric disturbances like scattering and turbulence effects. Surveillance tasks suffering from these limitations are airborne based reconnaissance, long range border control in rural areas and finally surface and submarine based naval observations.

Especially long observational paths through atmosphere closely to the sea surface are often limited by light scattering at water based aerosols in haze, mist or fog, depending on density and size of the aerosol droplets ${ }^{1}$. Since the scattering cross section for the relevant scattering mechanisms like Rayleigh or Mie are strongly dependent on the wavelength of the propagating light, it is a common method to overcome these limitations using imaging sensors sensitive in the infrared spectral range $^{2,3}$. The improvement in image contrast for far distant targets is twofold for longer wavelength ranges: In the first place the background scattering of the short wavelength based sunlight is eliminated, which typically puts a blue veil on top of the far distant daylight scene, thus reducing the target image contrast. Significant improvement can already be accomplished by use of long wavelength cut-on filters in the optical path to suppress the scattered short wavelength part of the sun light.

In the second place scattering of the reemitted sun irradiation of the target is responsible for the extinction of the object based light, finally entering the imaging sensor which is additionally reduced in contrast by the forward scattered light within the detected observation path. The images compared in figure 1 show, that the use of a SWIR sensor is already providing a large step towards increased far distant object contrast through haze compared to the strongly limited visual sight. The obvious benefit of the two thermal imagers, both sensitive at even longer wavelengths, is mainly 


\section{a. VIS camera}

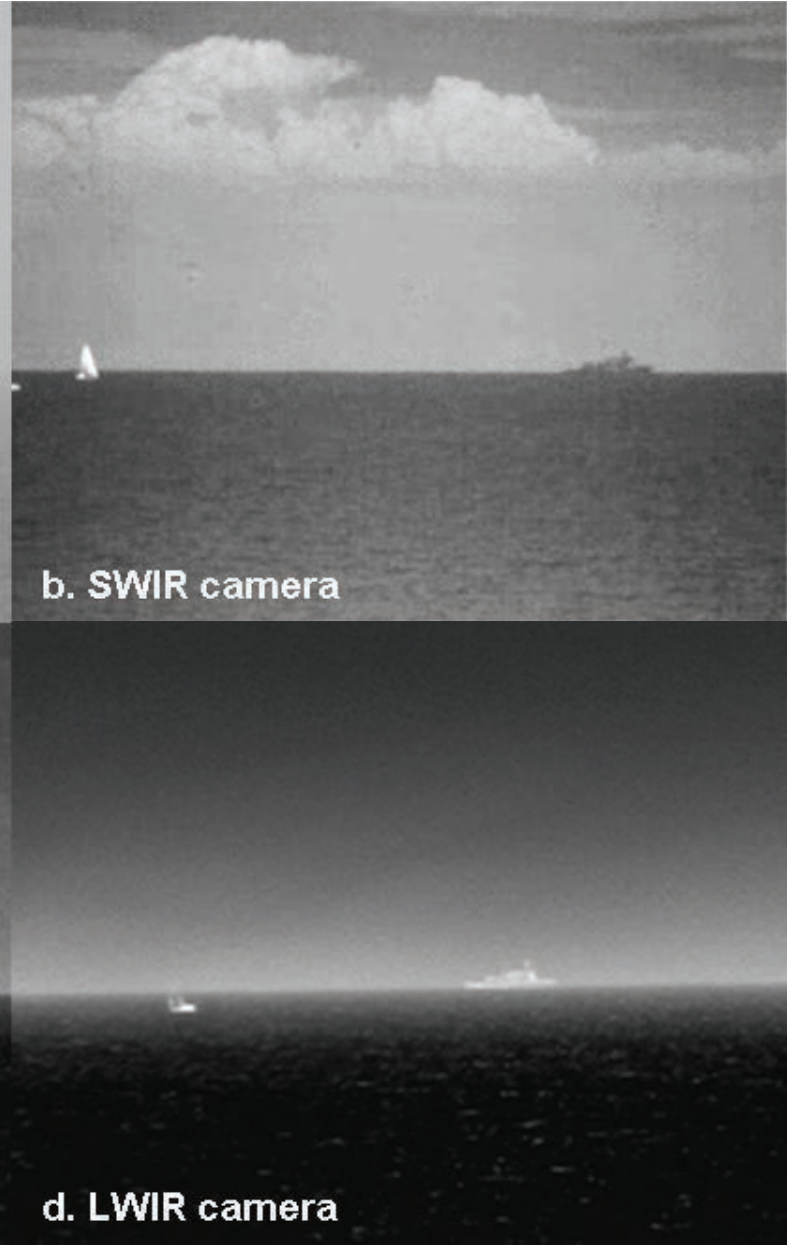

sensor platforms with required lowest possible geometrical signature. The shorter spectral waveband provides here e.g. for a so called attack optronics mast system (attack-OMS) a much smaller signature. High detection and identification capabilities are based on a single aperture achromatic continuous zoom lens from $2^{\circ}$ for the horizontal narrow field of view (NFOV), optional super- NFOV (SNFOV) of $1.5^{\circ}$ with $\mathrm{F} / 3.3$ for the visible path, up to $22^{\circ}$ wide field of view (WFOV) and simultaneous image acquisition in the visible and the SWIR. Both image streams can be used for further image processing like alpha blending or image fusion.

An integrated high extinction LRF enables the range determination of detected target objects. The system is intended to be used mainly at daylight conditions. No complete night vision capability is provided with this solution. Nevertheless the system is equipped with a so called night light detection (NLD) mode of the visible CMOS camera, that enables clear identification of colors from ship or harbor based position lamps in front of a dark background at night.

\section{Multispectral sensor suite description}

The multispectral sensor suite schematic is shown in figure 2. Along the zoom optical path 
the three branches for the relevant sensors HDTV, SWIR and LRF receiver diode are indicated to be supplied by the spectrally separated signals over the two beam splitters.

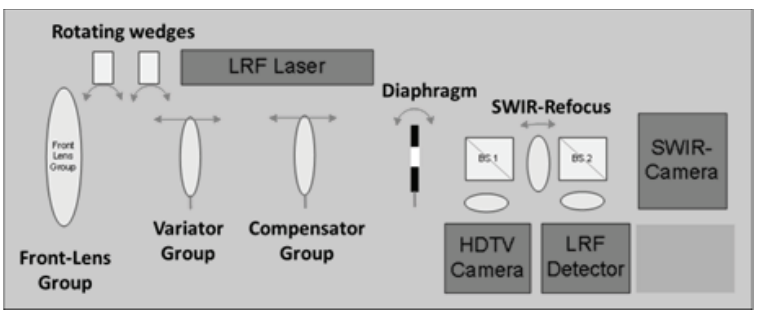

Fig. 1 Schematic layout of the multispectral sensor suite showing the main optical groups of the zoom optics with the motorized variator and compensator group, diaphragm, the so called 'SWIR-refocus' and the high reflective (HR) and high transmittance (HT) multispectral beam splitters, respectively. The LRF laser can be aligned by the indicated motorized wedges.

The HDTV CMOS sensor with $2.5 \mu \mathrm{m} \times 2.5 \mu \mathrm{m}$ increase the dynamic range of the sensor with respect to the available range of exposure times. The diaphragm is automatically adapted to avoid overexposure. The SWIR-camera video stream of the actual scenery is simultaneously available at a separate signal interface. Both images show the identical horizontal FOV (HFOV) along the same line of sight (LOS) with best focus in both optical channels.

The CMOS camera electronics offer additionally a long exposure detection mode, that enables exposure times of $t_{\text {exp }}>40 \mathrm{~ms}$, higher than the inverse frame rate. For a defined exposure time beyond the inverse frame rate, the integrated frames are displayed from internal memory at the current video frame rate.

An analogue back up mode based on analogue PAL or NTSC interlaced signal is supplied which can be displayed on a separate monitor

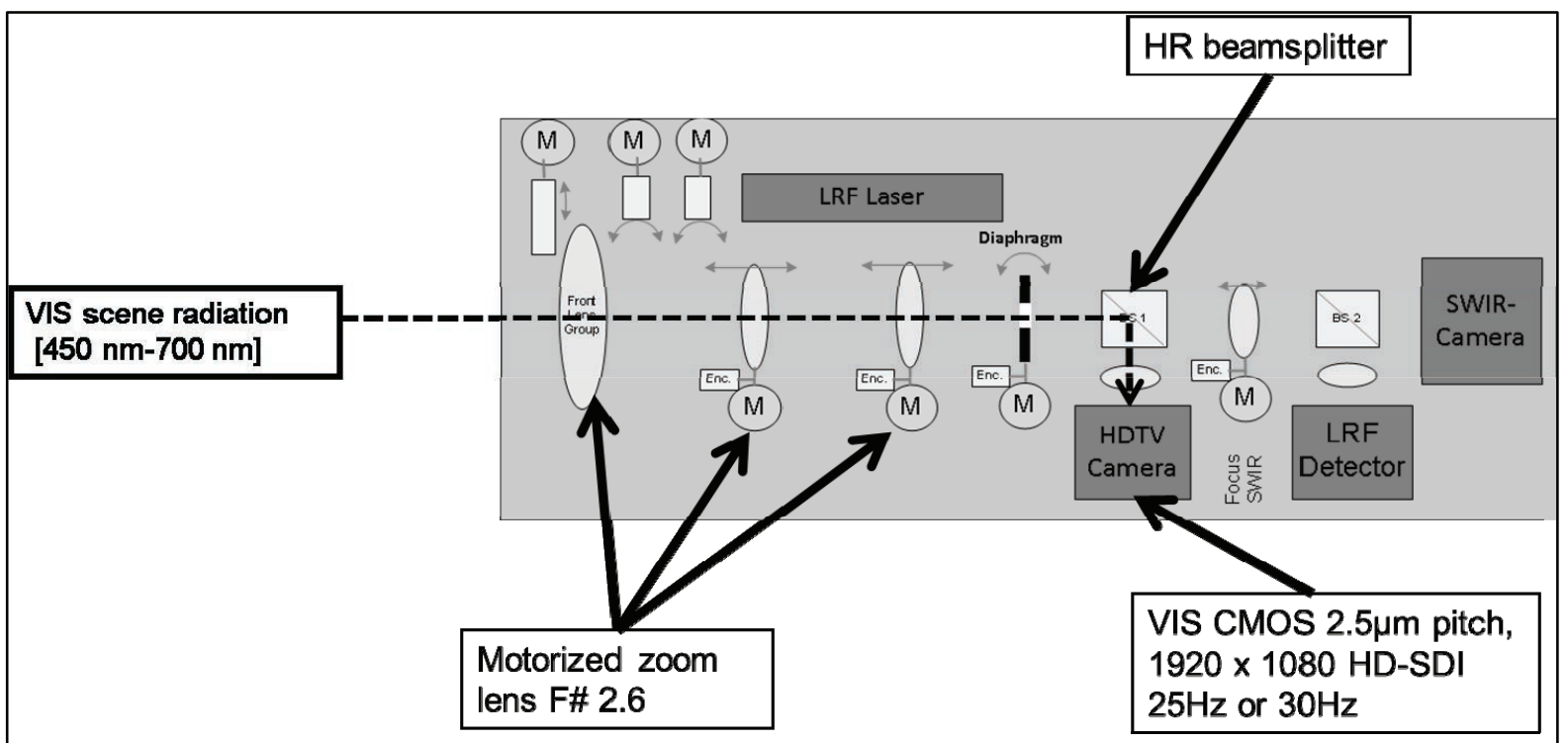

Fig. 2. Imaging path for the visual scene radiation through the front zoom lens group, spectrally separated from the remaining NIR and SWIR spectral part by a high reflective (HR) beam splitter.

pixel size and effective 1920 x 1080 pixel resolution is placed in the focal plane of a F/2.6 zoom lens with maximum focal length $f$ of $f_{\max }=$ $136.4 \mathrm{~mm}$. Continuous variation of the focal length down to $f_{\min }=12.3 \mathrm{~mm}$ results in a FOV range from $\mathrm{WFOV}=22^{\circ}$ down to $\mathrm{NFOV}=2^{\circ}$. At $\mathrm{F} / 3.3$ an optional SNFOV $=1.5^{\circ}$ with $\mathrm{f}_{\mathrm{SNFOV}}=$ $183 \mathrm{~mm}$ is available. The detailed signal and receiver path for the visible channel is shown in figure 2 .

The full HDTV resolution with either $25 \mathrm{~Hz}$ or 30 $\mathrm{Hz}$ frame rate is provided uncompressed at the signal interface of the sensor. An implemented diaphragm control mode can be activated to for the case that a digital image processing on boat level is temporarily not available.

The SWIR camera InGaAs FPA with $20 \mu \mathrm{m} x$ $20 \mu \mathrm{m}$ pixel size has a resolution of $640 \times 512$ pixels. A second version of an adapted rear refocus optics group is available for a smaller image plane corresponding to a pitch of $15 \mu \mathrm{m}$ with the image resolution of $640 \times 512$ pixel. The natively progressive image stream of the SWIR camera is up scaled within the camera video control (CVC) electronic to HD-SDI format by scaling the $640 \times 512$ resolution up to $1280 \times$ 1024 with a $2 x$ electronic zoom and embedding it into the $1920 \times 1080$ HD-SDI format. 


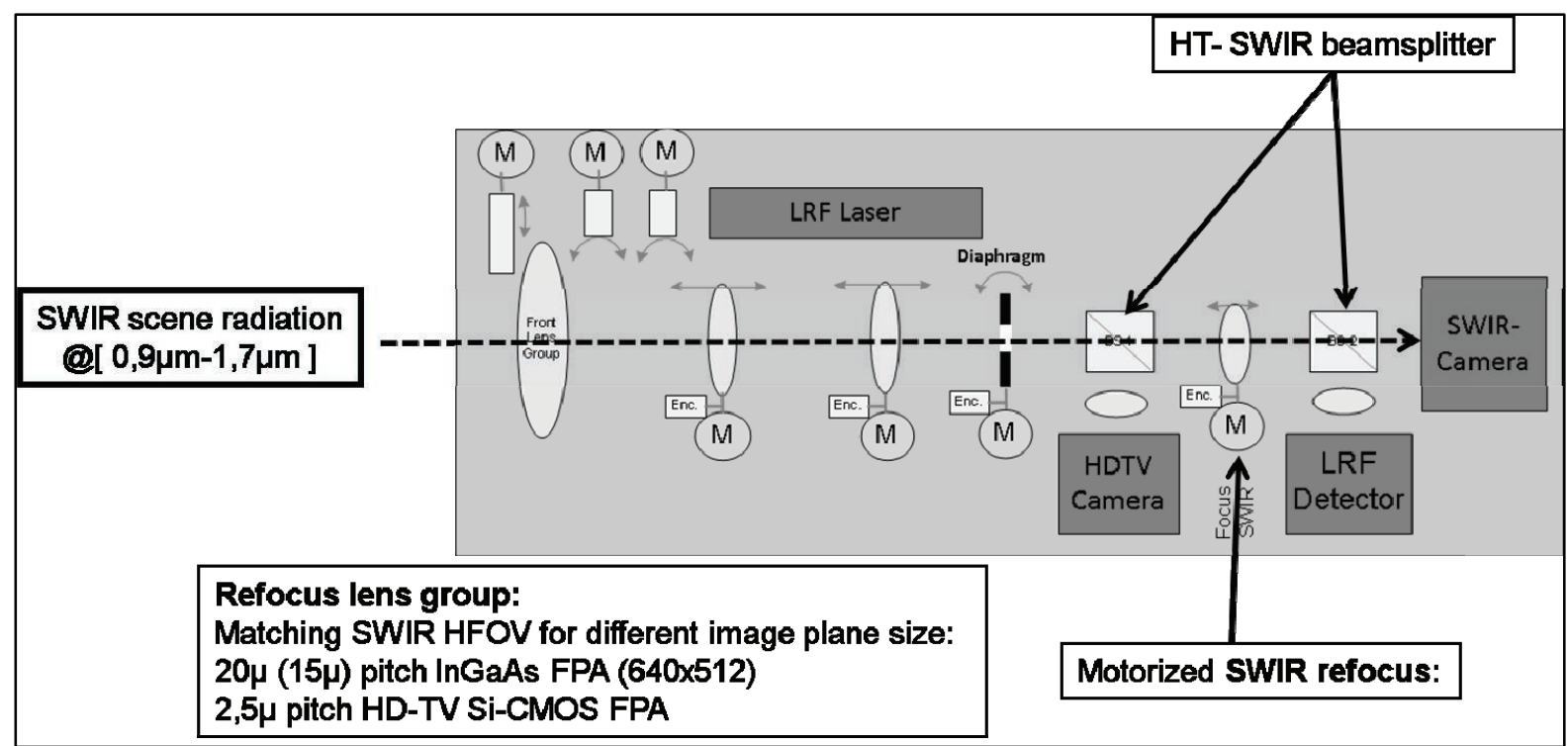

Fig. 3. Imaging path for the SWIR scene radiation through the front zoom lens group, the SWIR refocus group and the two high transmissible (HT) beam splitters.

To provide matched images from both cameras simultaneously in FOV and focus over the full zoom range the effective focal length for the SWIR imaging path is performed with the SWIR refocus group in figure 3 . alignment of the LRF transmission and receiver path relative to both imaging LOS's is performed with rotating wedges aligning a small portion of the emitted laser beam redirected within the so called laser harmonization path onto the SWIR FPA.

The schematic layout is shown in figure 5.

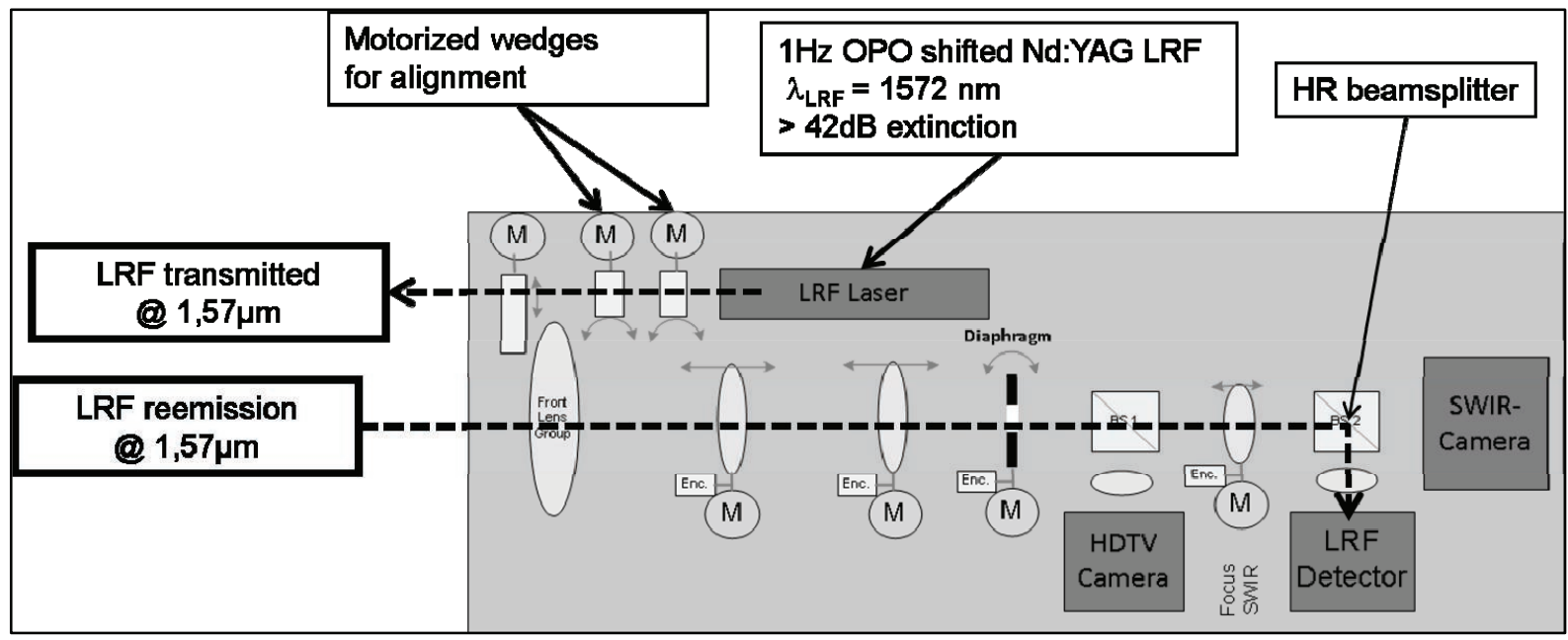

Fig. 4. Relevant beam and signal paths of the LRF transmitter and backscattered signal radiation , respectively. Receiver and transmitter LOS are exactly harmonized with the imaging sensors (HDTV camera for the visible (VIS) and and InGaAs camera for the SWIR).

Additionally the refocus group is moveable to correct sufficiently chromatic aberrations in the SWIR spectral range over the full zoom range.

The integrated LRF consists of a $1 \mathrm{~Hz}$ optical parametric oscillator (OPO) shifted YAG laser transmitter with $\lambda_{\mathrm{LRF}}=1572 \mathrm{~nm}$ primary emission and $>42 \mathrm{~dB}$ extinction. The LOS

\section{Optical design}

The optical design of the sensor is separated into the complete zoom based imaging optics and the LRF transmitter and harmonization path. The cross section of the imaging optics is shown in figure $6 a$ and $b$.

The front part of the optics up to the beam splitter 1 (BS1) consists of the front lens group, a variator and compensator group and a centering group. The front lens diameter is $55 \mathrm{~mm}$. In combination with the SWIR refocus lens group between BS1 and BS2 as seen in figure 6 , the effective focal lengths, F\# and corresponding horizontal FOV in both spectral 
channels are given for the NFOV and WFOV in table 1. In front of each focal plane (InGaAs and HDTV CMOS), a corresponding field lens group is necessary for best possible imaging quality on the FPA. In front of the HDTV CMOS camera a NIR-cut filter is located to guaranty best color fidelity.

In the LRF receiver channel the radiation is guided through the beam splitter BS2, partly reflecting the $1573 \mathrm{~nm}$ radiation with a bandwidth of $\pm \Delta \lambda=10 \mathrm{~nm}$ out of the whole SWIR scene radiation towards the LRF receiver optics and finally imaged onto the receiver diode with approximately $200 \mu \mathrm{m}$ spot diameter. The full achromatic design in both optical channels requires for best absolute transmission a corresponding broad band antireflection (AR) coating. An AR coating for all air/glass interfaces with lowest possible residual reflection between $450 \mathrm{~nm}$ to $1700 \mathrm{~nm}$ has been developed. In figure 7 design and measured spectrally resolved reflectivity on different test samples are compared. Special attention in the design of the AR coating has been spent on the best possible transmission for the LRF radiation, which directly influences its range performance.

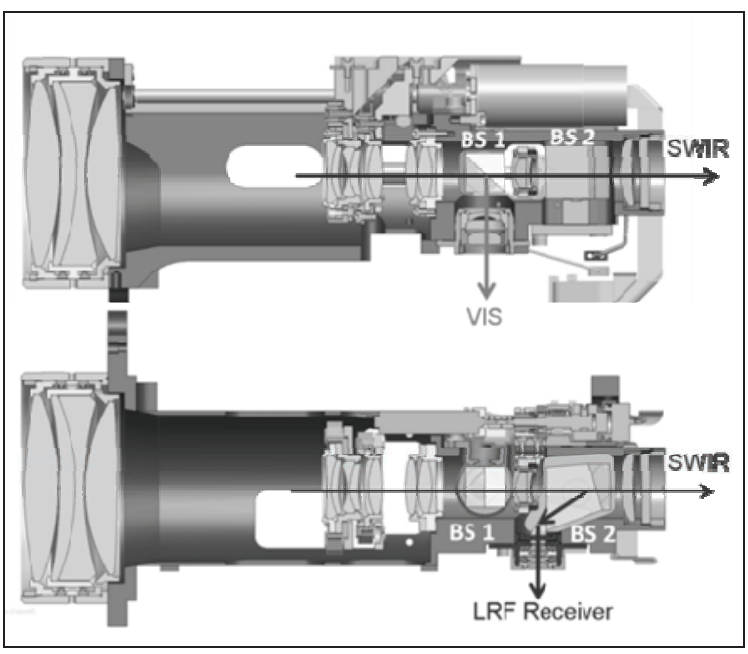

Fig. 6. Cross section of the imaging paths and LRF receiver for two orientations rotated by $90^{\circ}$ around the optical axis to visualize the two beam splitter paths BS1 and BS2. The lens group between BS1 and BS2 is the so called SWIR refocus group.

The most challenging optical components in the sensor design are nevertheless both beam splitters BS1 and BS2, shown in figure 6. The requirements on BS1 are crucial for either imaging performance in the VIS and SWIR optical path, respectively. Best possible reflectivity in the visual spectral part from $450 \mathrm{~nm}$ to at least $700 \mathrm{~nm}$ is required under a broad, asymmetric distribution of angular incidence from $35^{\circ}$ to $55^{\circ}$, accounting for the high zoom factor of $\times 11$ with a WFOV $=22^{\circ}$.
On the other hand highest possible transmission over the full SWIR spectral range is simultaneously required.

With the cube prism design the performance of our coating is shown in figure 8 with good results.

The results so far are very promising based on either image evaluation as well as on spectrally resolved reflectance and transmission measurements shown for $45^{\circ}$ beam incidence in figure 8 , representing the performance at the image center.

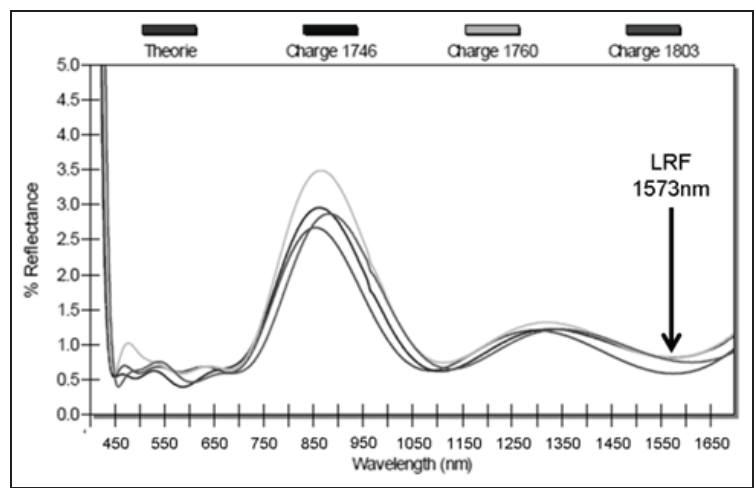

Fig. 7. Spectrally resolved residual reflectance of the broad band AR coating used for all glass/air interfaces in the sensors imaging optics. Measured values for three test charges are shown in comparison with design values. The arrow indicates the design based minimum reflectance for the LRF wavelength.

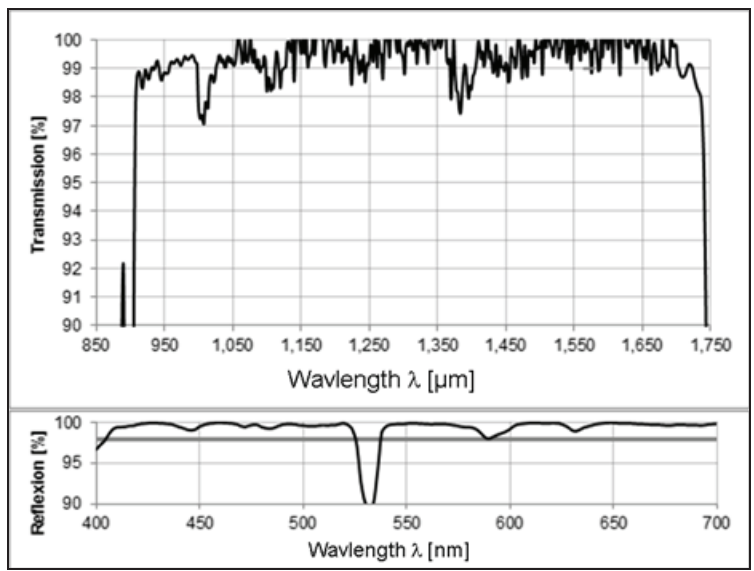

Fig. 8. Measured transmission (upper part for the SWIR) and reflectance (lower part for the VIS) of one test sample of our cemented cubical beam splitter BS1. The values have been corrected for the residual reflectance at the two air/glass interfaces.

The second beam splitter BS2 is responsible for simultaneously coupling out a narrow spectral band around the central LRF wavelength $\lambda_{\mathrm{LRF}}=$ $1.573 \mu \mathrm{m}$ and conducting it towards the receiver diode while additionally transmitting the whole SWIR spectral part of the scene based radiation onto the SWIR FPA. 
There are two variants implemented, resulting in different LRF range performance. In a first variant there is a $50 \%$ reflection coating with a bandpass centered at $1573 \mathrm{~nm} \pm 20 \mathrm{~nm}$ covering the complete beam splitter interface. The residual almost $50 \%$ radiation within $1573 \mathrm{~nm} \pm 20 \mathrm{~nm}$ is transmitted on the FPA of the SWIR camera. Based on this design, the total LRF extinction is at least $43 \mathrm{~dB}$. The requirement mentioned above is accomplished by a spatially limited highly reflective (HR) narrow band coating in the center of the imaging path footprint as shown in figure 9. As shown in the upper part of figure 9 the rays from the 2 mrad LRF FOV are reflected at the local HR coating in the prism interface. In the lower part of figure 9 the spectral ray distribution for the imaged SWIR radiation is shown. The local obstruction for the SWIR radiation around the LRF wavelength can result under low contrast scenes in a circular nonuniformity in the SWIR images which can be corrected by a scene based non-uniformity correction (NUC) in the SWIR camera.

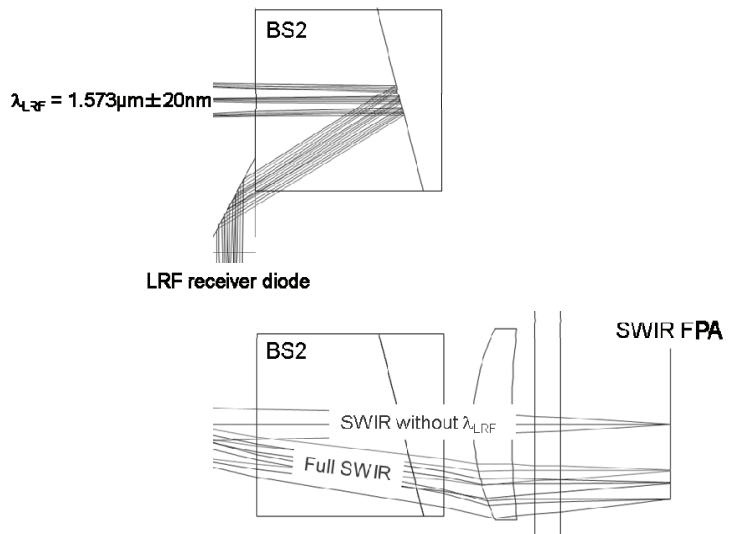

Fig. 9. Spectral and spatial separation of the LRF signal radiation from the imaged SWIR scene radiation on the SWIR FPA by beam splitter BS2in the high LRF extinction variant. The SWIR camera still receives the full SWIR spectrum of the scene.

\section{Mechanical Design}

The mechanical design of the combined sensor suite has been driven by the requirement to integrate all relevant components like optics, camera electronics, LRF, control and supply electronics and wiring looms into the limited space specified by the outline of the optronics mast head with less than $210 \mathrm{~mm}$ outer diameter. In figure 10 the complete system outline is shown. The outer dimensions of the complete module are given by a maximum diameter of $D=166.5 \mathrm{~mm}$ and a length of approximately $L=390 \mathrm{~mm}$. The total weight is less than $16 \mathrm{~kg}$. Due to the dissipated heat of all active components with an average total power dissipation of approximately
$\mathrm{P}_{\text {dissipation }}=25 \mathrm{~W}$ the main power consuming components like power supply units (PSU), FPGA-electronics and thermo-electrical cooler (TEC) of the SWIR camera are connected via heat pipes to an integrated heat exchanger.

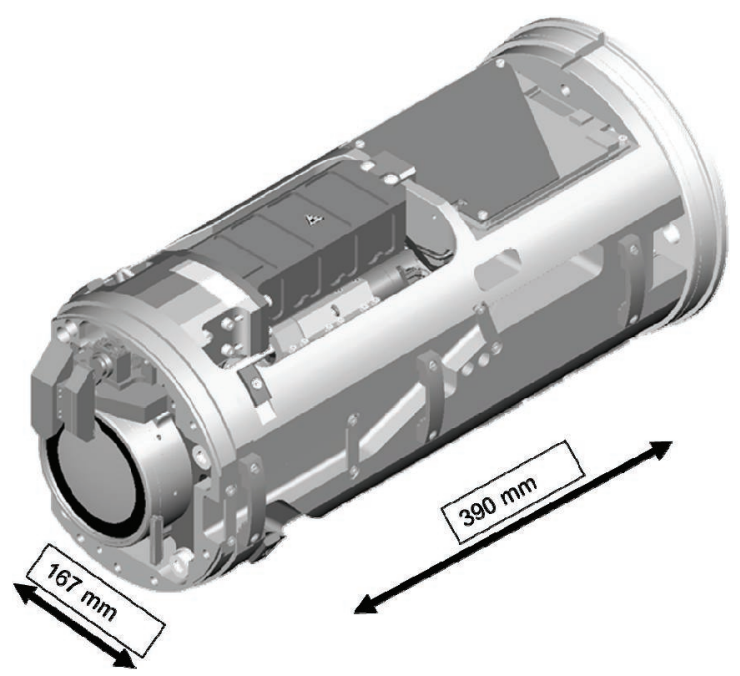

Fig. 10. Complete sensor suite mechanical outline and dimensions, including zoom optics, HDTV CMOS camera, SWIR camera, LRF transmitter and receiver, power supply units, video electronics and zoom lens control electronics.

\section{Electro-optical performances}

The combined modulation transfer function (MTF) determining the geometrical resolution on imager level is given by the product of the contributing MTF's.

$$
M T F_{\text {imager }}=M T F_{\text {optics }} \cdot M T F_{\text {detector }}
$$

For the visual and SWIR optical path the polychromatic on-axis MTF on imager level has been evaluated by Fourier transformation (FT) of the measured line spread function (LSF). Theoretical design values of the pure optical MTF are compared with the measured values for three spatial frequencies in four different FOV of table 1.

Comparing the MTF at the Nyquist frequencies of the diffraction limited optics with the MTF of the detector, both imaging paths in this sensor suite are detector pitch limited and given in table 1.

Besides the pure geometrical resolution the overall system sensitivity is mainly determined by the signal to noise ratio ( $\mathrm{S} / \mathrm{N}$ ) performance of the corresponding camera electronics, the F\# of the optics and the absolute transmission for either spectral range in the VIS and SWIR imaging paths.

The radiative transmission of the different imaging paths is depending on the quality of the 
optical coatings described in figure 7. Based on the measured transmission of the AR coatings and the beam splitter coatings we evaluated on system level the absolute transmission values as given in table 3 .

Tab. 1.: Comparison between theoretical MTF values of the corresponding optical channel and the measured MTF of the sensor on camera level with the 20um pitch SWIR camera. The corresponding optical design values for the new F/5.25 design in the SWIR imaging path is also given. measurements at $15^{\circ} \mathrm{C}$ FPA temperature on representative units used in the system.

In accordance with table 4 the CMOS camera used in the sensor suite has a quite low noise level offering relatively good low light sensitivity, especially in combination with the fast optics in the VIS channel. The used VIS CMOS camera offers a dynamic range of $>70 \mathrm{~dB}$ based on the parameters listed in table 4.

\begin{tabular}{|c|c|c|c|c|c|c|}
\hline MTF VIS imager & $\begin{array}{l}\text { MTF }_{\text {optics }} \\
\text { optical design }\end{array}$ & $\begin{array}{l}\text { MTF }_{\text {imager }} \\
\text { measured }\end{array}$ & MTF SWIR imager & $\begin{array}{l}\text { MTF }{ }_{\text {optics }} \\
\text { optical design } \\
\text { F/7.0 }\end{array}$ & 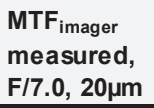 & $\begin{array}{l}\text { MTF } \text { optics } \\
\text { optical design } \\
\text { F/5.25 }\end{array}$ \\
\hline MTF & & & MTF & & & \\
\hline $40 \mathrm{LP} / \mathrm{mm}$ & & & $4 \mathrm{LP} / \mathrm{mm}$ & & & \\
\hline $\operatorname{NFOV}\left(2,0^{\circ}\right)$ & $76 \%$ & $80 \%$ & $\operatorname{NFOV}\left(2,0^{\circ}\right)$ & $93 \%$ & $93 \%$ & $87 \%$ \\
\hline MNFOV $\left(4,0^{\circ}\right)$ & $79 \%$ & $84 \%$ & $\operatorname{MNFOV}\left(4,0^{\circ}\right)$ & $91 \%$ & $96 \%$ & $95 \%$ \\
\hline MFOV $\left(12^{\circ}\right)$ & $83 \%$ & $83 \%$ & MFOV $\left(12^{\circ}\right)$ & $90 \%$ & $93 \%$ & $94 \%$ \\
\hline WFOV $\left(22^{\circ}\right)$ & $81 \%$ & $82 \%$ & WFOV $\left(22^{\circ}\right)$ & $90 \%$ & $90 \%$ & $94 \%$ \\
\hline MTF & & & MTF & & & \\
\hline $80 \mathrm{LP} / \mathrm{mm}$ & & & $8 \mathrm{LP} / \mathrm{mm}$ & & & \\
\hline $\operatorname{NFOV}\left(2,0^{\circ}\right)$ & $48 \%$ & $45 \%$ & NFOV $\left(2,0^{\circ}\right)$ & $86 \%$ & $74 \%$ & $67 \%$ \\
\hline MNFOV $\left(4,0^{\circ}\right)$ & $51 \%$ & $54 \%$ & $\operatorname{MNFOV}\left(4,0^{\circ}\right)$ & $78 \%$ & $84 \%$ & $87 \%$ \\
\hline MFOV $\left(12^{\circ}\right)$ & $62 \%$ & $52 \%$ & $\operatorname{MFOV}\left(12^{\circ}\right)$ & $75 \%$ & $78 \%$ & $84 \%$ \\
\hline WFOV $\left(22^{\circ}\right)$ & $60 \%$ & $54 \%$ & WFOV $\left(22^{\circ}\right)$ & $74 \%$ & $70 \%$ & $84 \%$ \\
\hline MTF & & & MTF & & & \\
\hline $160 \mathrm{LP} / \mathrm{mm}$ & & & $24 \mathrm{LP} / \mathrm{mm}$ & & & \\
\hline $\operatorname{NFOV}\left(2,0^{\circ}\right)$ & $26 \%$ & $20 \%$ & NFOV $\left(2,0^{\circ}\right)$ & $57 \%$ & $12 \%$ & $22 \%$ \\
\hline $\operatorname{MNFOV}\left(4,0^{\circ}\right)$ & $25 \%$ & $20 \%$ & $\operatorname{MNFOV}\left(4,0^{\circ}\right)$ & $36 \%$ & $19 \%$ & $58 \%$ \\
\hline $\operatorname{MFOV}\left(12^{\circ}\right)$ & $37 \%$ & $20 \%$ & $\operatorname{MFOV}\left(12^{\circ}\right)$ & $30 \%$ & $23 \%$ & $42 \%$ \\
\hline WFOV $\left(22^{\circ}\right)$ & $38 \%$ & $21 \%$ & WFOV $\left(22^{\circ}\right)$ & $30 \%$ & $15 \%$ & $37 \%$ \\
\hline
\end{tabular}

Tab. 2. Comparison between cut-off MTF frequencies of either spectral channel, VIS and SWIR respectively for the limiting case of diffraction limited optics and squared pitch based MTF. Both spectral paths are detector limited by design.

\begin{tabular}{|c|c|c|}
\hline & 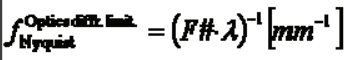 & $f_{\text {Nyquint }}^{\text {detoctor }}=d^{-1}\left[\mathrm{~mm}^{-1}\right]$ \\
\hline VIS F/2.6 & $712.25[@ 540 \mathrm{~nm}]$ & 400.0 \\
\hline SWIR FI.0 & 95.24 [@1500nm] & 50.0 \\
\hline SWIR F/5.25 & 126.98 [@1500nm] & 66.67 \\
\hline
\end{tabular}

Tab. 3.Calculated radiative transmission in the two imaging paths based on spectrally resolved measurements on AR and beam splitter coatings.

\begin{tabular}{|l|c|}
\hline & Transmission \\
\hline VIS & $>75 \%$ \\
\hline SWR & $>65 \%$ \\
\hline
\end{tabular}

The $\mathrm{S} / \mathrm{N}$ of the cameras are determined by the spectrally dependent quantum efficiency (QE), the detector noise such as the read out noise and the dark current based shot noise. The parameters listed below are all based on
The SWIR camera electronics contributes significant noise electrons under low signal conditions. The noise equivalent irradiance (NEI) of the SWIR sensor is given at different levels of the optical path hereafter. The noise equivalent irradiance on FPA level is given by

$$
N E I_{\text {Photon }}=\frac{\sqrt{R N^{2}+D C \cdot t_{\exp }}}{Q E \cdot A_{d} \cdot t_{\exp }}
$$

$N E I_{\text {Photon }}=$ noise equivalent

irradiance on FPA level[photons $/ \mathrm{sec} \cdot \mathrm{cm}^{2}$ ]

with $R N=$ read out noise, $t_{\exp }=$ exposure time

$D C=$ dark current, $A_{d}=$ detector area,

$Q E=$ quantum efficiency

The equivalent irradiance in terms of power is given by: 
$N E I_{E}=N E I_{\text {Photon }} \cdot \frac{h \cdot c}{\lambda}$

$N E I_{E}=$ noise equivalent

irradiance on FPA level [W / $\mathrm{cm}^{2}$ ]

with

$h=6.626 \cdot 10^{-34} \mathrm{~J} \cdot \mathrm{s}, c=3.0 \cdot 10^{8} \mathrm{~m} \cdot \mathrm{s}^{-1}$

$\lambda=1.5 \mu \mathrm{m}$

Table 5 gives the corresponding NEI values for the different SWIR sensors according to equation (2) and (3).

The NEl on target level depends on the absolute transmission and the F\# of the optics and is calculated for an extended radiation source by

$N E I_{T \text { arg } e t}=\frac{E_{\text {Photon }} \cdot\left(4 \cdot F \#^{2}+1\right)}{\pi \cdot \tau} \cdot \frac{h \cdot c}{\lambda}$

with

$F \#=\mathrm{F}$ - number of optics

$\tau=$ transmission of optics at $\lambda$

Tab. 4. S/N determining parameters of the used

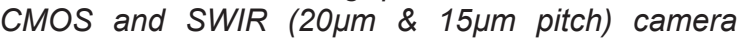
electronics.

\begin{tabular}{|c|c|c|c|}
\hline & $\begin{array}{c}\text { Quantum Efficiency, } \\
\text { QE }\end{array}$ & $\begin{array}{l}\text { Read } \\
\text { out } \\
\text { noise, } \\
R N\end{array}$ & $\begin{array}{l}\text { Dark current } \\
\text { DC }\end{array}$ \\
\hline $\begin{array}{l}\text { VIS } \\
\text { CMOS }\end{array}$ & $\begin{array}{l}45 \% \\
\text { (@540nm) }\end{array}$ & $5 \mathrm{e}^{-}$ & $0.08 \mathrm{e}-/ \mathrm{s}$ \\
\hline $\begin{array}{l}\text { SWIR } \\
20 \mu \mathrm{m} \\
\text { pitch }\end{array}$ & $\begin{array}{l}70 \% \\
\text { (average } 0.9 \mu \mathrm{m}-1.7 \mu \mathrm{m} \text { ) }\end{array}$ & $121 \mathrm{e}^{-}$ & $2.4 \mathrm{E}+5 \mathrm{e}^{-} / \mathrm{s}$ \\
\hline $\begin{array}{l}\text { SWIR } \\
15 \mu m \\
\text { pitch }\end{array}$ & $\begin{array}{l}75 \% \\
\text { (average } 0.9 \mu \mathrm{m}-1.7 \mu \mathrm{m} \text { ) }\end{array}$ & $40 \mathrm{e}^{-}$ & $1.34 \mathrm{E}+4 \mathrm{e} / \mathrm{s}$ \\
\hline
\end{tabular}

Table 5: NEI of the SWIR detectors based on noise and system parameters given in tables 4,3 and 2 and equations (2), (3) and (4).

\section{Conclusions}

The properties and capabilities of a highly compact multispectral sensor suite combined with a high extinction LRF harmonized in all line of sights provide HD-SDI based images of the SWIR and VIS camera and range finding capabilities. The imaging sensors match in focus and FOV over the full zoom range, further image processing capabilities like alpha blending and pyramid image fusion are supported for advanced detection and recognition tasks.

The compact design of the sensor suite is reached by a combined optical design with only one common entrance aperture for all three sensors, the SWIR camera, the HDTV CMOS camera and the laser receiver, separating spectrally the different wavelength ranges for the sensors. In this combination the diameter of the sensor suite is mainly defined by the diameter of the optics. The laser emitter is separated but small compared to the receiver optics. The electro-optical design has been measured and discussed as well as the quality of the compact and complex optical paths. The electro-optical performances of the different SWIR spectral channel designs have been compared. Limited twilight sensitivity of the SWIR sensor is reached with the estimated NEI on target level of approx. $1,7{ }^{*} 10^{11}$ photons / $\left(\mathrm{sec}^{*}\right.$ sterad $\left.{ }^{*} \mathrm{~cm}^{2}\right)$.

\begin{tabular}{|l|c|c|c|}
\hline $\begin{array}{l}\text { NEI (15 } \\
\lambda=1.5 \mu \mathrm{C} \text { FPA, }\end{array}$ & $\begin{array}{l}\text { Nolse equlvalent photons } \\
\text { on FPA level, equ.(2) }\end{array}$ & $\begin{array}{l}\text { Nolse equlvalent } \\
\text { irradiance on FPA level, } \\
\text { equ.(3) }\end{array}$ & $\begin{array}{l}\text { Nolse equlvalent photon radlance of extended } \\
\text { target, equ.(4) }\end{array}$ \\
\hline & $\begin{array}{l}\text { [photons' } \\
\left.\text { sec } \times \mathrm{cm}^{2}\right]\end{array}$ & [W/cm²] & $\begin{array}{c}\text { [photons' } \\
\left.\text { sec } \times \text { sterad } \times \mathrm{cm}^{2}\right]\end{array}$ \\
\hline $\begin{array}{l}20 \mu \mathrm{m} \text { pitch } \\
\text { InGaAs }\end{array}$ & $1.64 \mathrm{E}+9$ & $2.17 \mathrm{E}-10$ & $1,71 \mathrm{E}+11$ \\
\hline $\begin{array}{l}15 \mu \mathrm{m} \text { pitch } \\
\text { InGaAs }\end{array}$ & $8.84 \mathrm{E}+8$ & $1.17 \mathrm{E}-10$ & $5.17 \mathrm{E}+10$ \\
\hline
\end{tabular}




\section{References}

[1] Martin Gerken, Bertram Achtner, Michael Kraus, Tanja Neumann, Mario Münzberg, "Shortwave infrared camera with extended spectral sensitivity". Proc SPIE 8353-2 (2012)

[2] M. Hübner, M. Gerken, B. Achtner, M. Münzberg, "Multispectral SWIR/VIS continuous zoom camera with integrated laser range finder based on single optical channel design for future submarine optronics mast applications", OPTRO-2014$2934429(2014)$

[3] M. Hübner, M. Gerken , B. Achtner, M. Kraus , M. Münzberg, "Compact multispectral continuous zoom camera for color and SWIR vision with integrated laser range finder", Proc SPIE 9070-64 (2014) 